\title{
Preclinical Evaluation of Anti-HIV-I Polybiguanide Vaginal Microbicides in a Murine Model of Toxicity and Inflammation Tina Kish-Catalone* ${ }^{* 1}$, Mohamed E Labib ${ }^{2}$, Robert F Rando ${ }^{2}$, Brian Wigdahl ${ }^{1}$ and Fred C Krebs ${ }^{1}$
}

Address: ${ }^{1}$ Department of Microbiology and Immunology, and Institute for Molecular Medicine and Infectious Disease, Drexel University College of Medicine, Philadelphia, PA, USA and ${ }^{2}$ Novaflux Biosciences, Inc., Princeton, NJ, USA

* Corresponding author $¥$ Presenting author

from 2005 International Meeting of The Institute of Human Virology

Baltimore, USA, 29 August - 2 September 2005

Published: 8 December 2005

Retrovirology 2005, 2(SuppI I):S98 doi:10.1186/1742-4690-2-SI-S98

The use of vaginal microbicides has gained support as a strategy for the protection of women against HIV-1 and other sexually transmitted disease (STD) pathogens. The preclinical Swiss Webster murine model has been developed to specifically measure cervicovaginal tissue integrity and inflammation following application of candidate vaginal microbicides, when potential exposure to an STD pathogen may occur. This model demonstrates both mechanistic and temporal differences in inflammatory responses following microbicide exposure. Currently, specific markers of inflammation, including pro-inflammatory cytokines, are being evaluated in the cervicovaginal mucosa. Safety profiles of polybiguanides (PBGs), which demonstrated significant in vitro efficacy against HIV-1, are being assessed in vivo. Intravaginal application of PEHMB $(1 \%)$ resulted in little or no cervicovaginal toxicity after short- or long-term exposure. Collectively, these studies support the Swiss Webster model as a valuable tool for the preclinical assessment of toxicity and inflammation associated with exposure to candidate topical microbicides. Furthermore, these results strongly support further development of polybiguanide derivatives as vaginal microbicidal agents. 